\title{
Rabies, the neglected cause of mortality in developing countries
}

DMSJ;19(2) 13-16

http://dx.doi.org/10.4314/dmsj.v19i2.4

Kessy Immaculata P.\{MD3\}

Institution: Muhimbili University of Health and Allied Sciences (MUHAS)

\begin{abstract}
BACKGROUND:Rabies is a very deadly zoonosis, caused by a lyssavirus which belongs to the family Rhabdoviridae. The reservoirs include wild wolves, bats, skunks, raccoons and foxes. This disease satisfies all World Health Organization (WHO) criteria for diseases that are a priority for control. Contrary to expectations, its control is unfortunately neglected especially in developing countries. Rabies is recognized as one of the causes of many preventable deaths. Globally it causes 35,000 deaths annually and strikingly, 24,000 of the deaths occur in Africa. A multi-centre study from India reported 18,500 human rabies deaths per year, while in Tanzania 380-1,900 deaths have been reported to occur annually. Most of the studies on rabies in Tanzania have been conducted by foreign scientists and the WHO and few Tanzanians-often junior researchers and research assistants. Currently, there is an on-going study at Ifakara on immunization of dogs and results are still pending. Rabies is a fatal disease that carries a very high mortality. Tanzanians are encouraged to carry out several studies in different regions especially in rural areas and congested urban areas where stray dogs are plenty so as to combat this deadly disease. There is paucity of information regarding the actual magnitude of this disease in developing countries including Tanzania, resulting in a gap that needs to be filled by conducting research on the subject. Scientists in Tanzania are therefore encouraged to carry out several studies in different regions especially in rural areas and congested urban areas where stray dogs are in plenty so as to generate comprehensive information about rabies that will contribute to the control and prevention of this deadly disease.
\end{abstract}

MAJOR FINDINGS: The global DALY (Disability Adjusted Life Years) value is about 1.16 million that is much greater than that of a disease like leprosy that has a DALY value of about 117,000 , but the latter has been given prominence as a disease of public health importance. This observation signifies the need to promote public awareness regarding rabies and also the need to implement workable and effective interventions for control and prevention. After a review of different articles on this topic, it is evident that rabies affects children, with a prevalence of $26 \%$ among those aged between 0-4 years and 34\% among those aged 5-14 years. Prevalences of 33\%, 3.5\% and $4.2 \%$ were reported among individuals aged 15-44 years, 45-59 years, and 60 years and above, respectively. Causes of neglect of this disease include lack of political commitment towards control of the disease, low awareness about the magnitude of the problem due to under reporting of cases, high cost of Post-Exposure Prophylaxis, low coverage of vaccination of dogs in developing countries and lack of proper ownership and care for dogs that turn out to be 'stray dogs' that serve as reservoirs for the infection.

CONCLUSION: Rabies has caused significant preventable mortality in developing countries and affects mostly the juvenile population. Domestic dogs should be well vaccinated and reached with veterinary services, there should be a joint venture among the political leaders, health care providers, veterinary doctors and the mass at large to control this disease. There should be thorough mass education and facilitation to community participation. PEP should be available in close proximity so aiming at reducing mortality in population groups at high risk of infection.

Correspondence to: Kessy Immaculata P.,

Muhimbili University of Health and Allied Sciences;

E-mail:immacuiatakessy@yahoo.com

\section{INTRODUCTION}

Rabies is a viral zoonosis caused by negative-stranded RNA viruses from the Lyssavirus genus. Genetic variants of the genotype 1 Lyssavirus (the cause of classical rabies) are maintained in different parts of the world by different reservoir hosts within 'host-adaptive landscapes' (1). Although rabies can infect and be transmitted by a wide range of mammals, reservoirs comprise only mammalian species within the Orders Carnivora (e.g. dogs, raccoons, skunks, foxes, jackals) and Chiroptera (bats). Rabies is often perceived as a rare or insignificant disease of humans in developing countries, which perception has been a major factor hampering development of disease control initiatives (2). While the control of this disease needs joint venture, majority leave this role upon the veterinary authorities ${ }^{(2)}$. Rabies control has been successful in developed countries due to multidisciplinary participation in its control, dog 
vaccination and detaining of domestic dogs. The condition of rabies control in developing countries especially in Africa and Asia is not yet satisfactory; there's still a lot to be done in obtaining data on cases of dog bites, morbidity post- rabid dog bites and mortality due to rabies ${ }^{(3)}$.

Mortality is contributed to by inaccessibility to post exposure prophylaxis (PEP) especially for those staying far away from health centers, and poor infrastructure causing death to some on their way to hospital ${ }^{(5)}$. Lack of government policy on combating this disease makes poor countries lose their man power as adolescents and youths are mostly affected.

\section{METHODOLOGY}

Several articles that have addressed this problem were reviewed in order to assess the extent of neglect, the epidemiology of rabies in the developing and developed world, to determine the disparity between the developed and developing world and to deduce possible strategies for implementing available interventions for control and prevention. The review was mainly based on studies done in the developing world as there were few studies conducted in developed countries for comparison.

As much as possible, old references were excluded except for those of historical interest. Also, limited attention was given to those based purely on the epidemiological pattern of rabies in western countries because they were considered inadequate to portray the real situation in developing countries.

\section{PRESENTATION OF DATA}

From the various studies conducted, the reported burden of rabies is variable from $26 \%$ among children aged $0-4$ years; $34 \%$ among those aged 5-14 years; $33 \%$ among the 15-44 year olds; $3.5 \%$ among those aged between 45-59 and $4.2 \%$ among those aged 60 years and above ${ }^{(2)}$.

Several studies have been conducted to determine the mortality due to rabies including calculation of Disability Adjusted Life Years (DALY). The DALY is a standardized, comparative measure of disease impact developed to assess the relative impact of different diseases across different settings and at different stages of economic and public health development ${ }^{(7)}$. The DALY is a combination of the years of life lost (YLL) due to premature death and the years of life lived with a disability (YLD).

The global DALY for rabies is based on annual WHO estimates of 35,000 deaths. This only includes the YLL but does not include YLD, hence the estimated DALY for the above figure is 1.16 million ${ }^{(2)}$. Graphical representations below show how rabies causes the highest mortality overall in comparison with other zoonoses and has a relatively high DALY compared to other diseases like lymphatic filariasis, schistosomiasis, Chaga's disease and leishmaniasis that are regarded as neglected diseases of public heath importance.

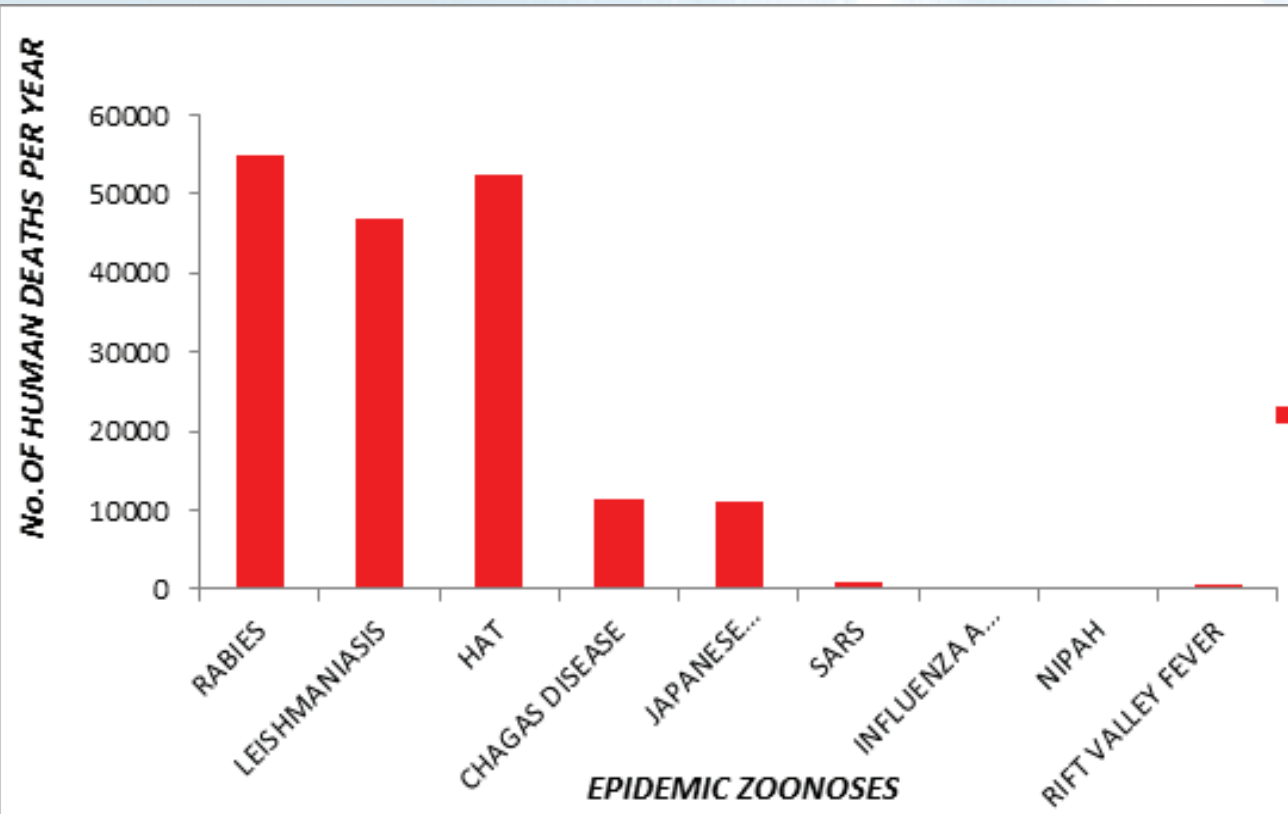

Figure 1: Numbers of human deaths per year for rabies compared with peak annual deaths from selected epidemic zoonoses

Data source: (Severe Acute Respiratory Syndrome, SARS, 2003; H5N1, 2006; Nipah, 1999; and Rift Valley Fever 2007). Data sources: Rabies (Ref 13), Leishmaniasis, Human African Trypanosomiasis (HAT), Chagas Disease and Japanese Encephalitis (Ref 14), SARS (Ref 15), Influenza A H5N1 (Ref 16), Nipah (Ref 17), Rift Valley Fever (Ref 18,Ref 19). 


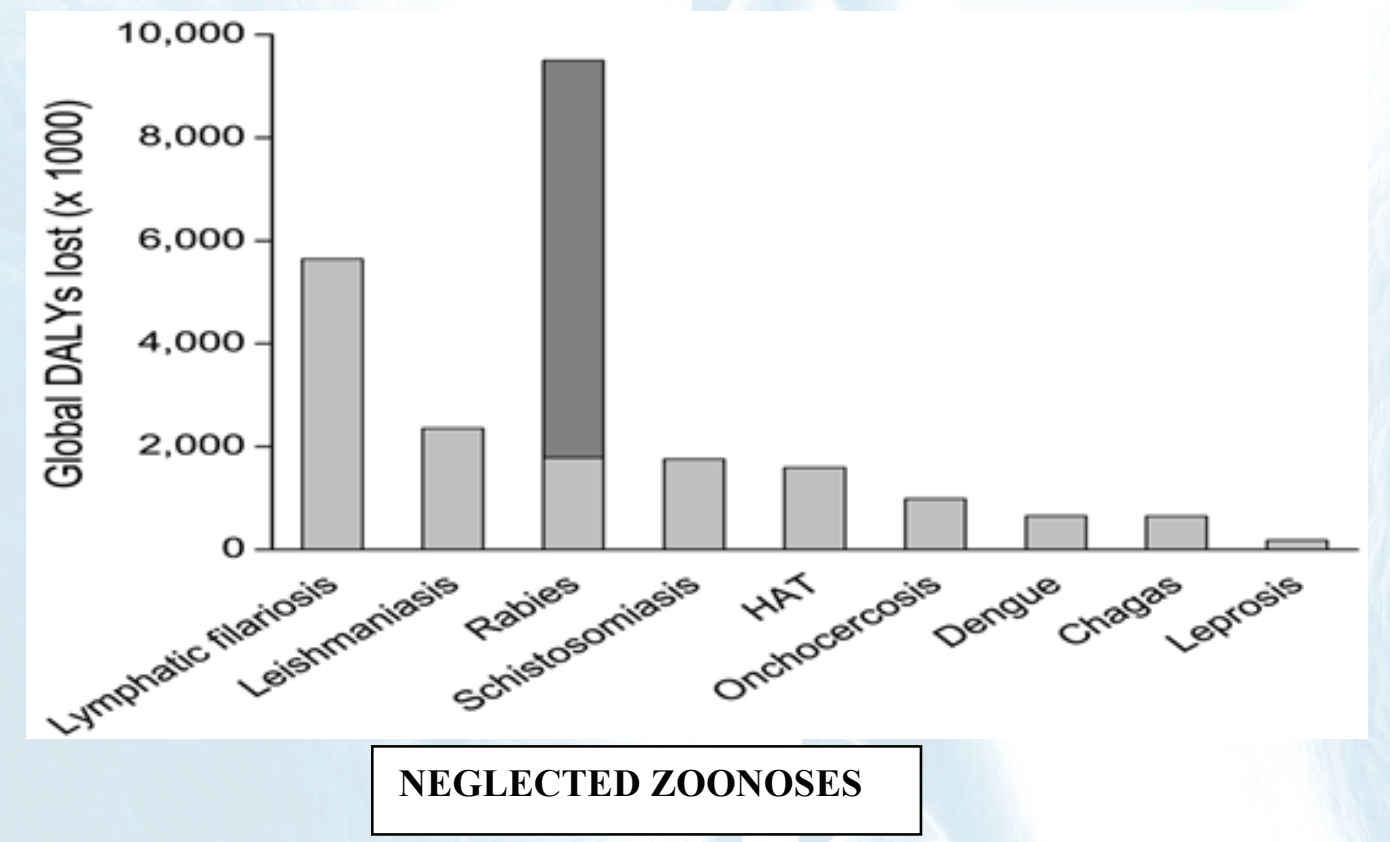

Figure 2: Global DALY scores for neglected tropical diseases reported assuming no post-exposure treatment (dark grey). Data source (Ref 2)

There are many causes of neglecting canine rabies control in developing countries including under-estimation of the problem due to under-reporting of rabid canine attacks caused by:

- Rabies victims being too ill to go to hospital or dying on the way before reaching hospital.

- Some superstitions and beliefs linking rabies patients' symptoms to witchcraft especially in rural areas

- Clinically diagnosed cases may go unreported to the central authorities due to lack of political interest in rabies control

- Misdiagnosis of some rabies cases; for example in Malawi 11.5\% of cases diagnosed as cerebral malaria were proved wrong at autopsy ${ }^{(8)}$

Another bottle-neck to combating this disease in developing countries is lack of knowledge of ownership of 'stray dogs' and the dog population overall. In Tanzania, a mark-recapture method has been used to ascertain the dog population in relation to their ownership. It was established that very few dogs are ownerless; hence the presence of many stray dogs is likely due to unwillingness or inability of owners to confine them ${ }^{(9)}$.

Economically, Post-Exposure Prophylaxis (PEP) is very expensive and inaccessible in many cases. Most patients die on their way to hospital due to long distances before they get PEP ${ }^{(1)}$. Many countries in Asia, such as Thailand, Vietnam and Sri Lanka have greatly reduced human rabies deaths through increased PEP use, but at very high cost ${ }^{(10)}$.

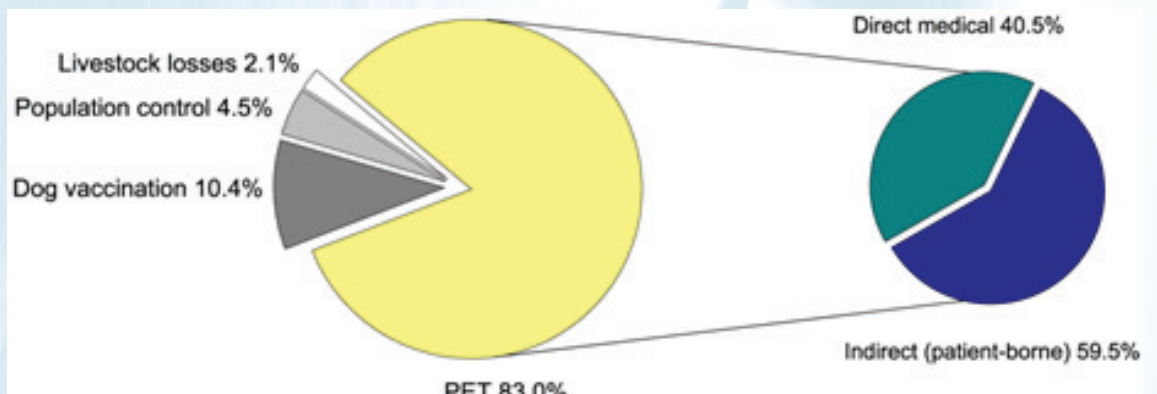

Figure 3: Economic burden of canine rabies (data source: ref 1).

Vaccination of dogs is a method thought to be very helpful in rabies control and cost effective as shown in the figure above. However, widespread rabies vaccination campaigns have been difficult to implement due to unclear dissemination of information as well as improper ownership of dogs ${ }^{(11)}$.

\section{DISCUSSION}

Many studies conducted have proved that rabies is fatal but neglected, some emphasizing the use of PEP, others advocating vaccination as the proper control measure and some accepting both. The main gap still remains in defining the magnitude and impact of the problem, and educating the community about the problem so as to improve control and prevention. Apart from the reported misdiagnosis in Malawi, this shortcoming has not been reported by other countries, leading to speculations about what might be happening in some hospitals in Tanzania as far as rabies diagnosis is concerned. One is left to wonder whether there is correct diagnosis at all stages of health care in Tanzania. Regarding the role of taking initiatives in combating the problem, this should not be left as a health sector issue only but other complementary initiatives should also be pursued including political initiatives. The diagnostic criteria used to diagnose rabies appear to be 
inadequate in some situations. Additionally, much still has to be done on researches concerning rabies, preferably through collaborative multidisciplinary joint ventures so as to generate evidence-based strategies for eradication of rabies. In this context, the One Health initiative which is now been promoted in Tanzania and elsewhere in Africa provides an appropriate platform for addressing multisectoral response to rabies.

\section{CONCLUSION}

Rabies has caused a huge economic burden in developing countries and affects mostly the juvenile population ${ }^{(2)}$. It is recommended to promote vaccination of domestic dogs which should also be reached with veterinary services. There should be a joint venture among the political leaders, health care providers, veterinary doctors and the community at large to control this disease. Mass education and facilitation of community participation should be enhanced. PEP should be available in close proximity to where people live and work so as to save the lives of rabid dog bite victims.

\section{ACKNOWLEDGEMENTS}

I thank almighty God for guiding me through. Prof E. Lyamuya and Prof. M.I.N. Matee helped me review my article. I thank my friends like Simon Mushi, Glory Msacky, Chai Chaita, Canuty Fides, Ngoitanile Elizabeth, and Mbwanji Gladys; Father Sylvester and Deomedi; and my family for moral support.

God bless you.

\section{REFERENCES}

1. Lembo T, Hampson K, Kaare MT, et al. The feasibility of canine rabies in Africa: Dispelling doubts with data, Plos Negl tropical diseases 2010; 4(2):e626

2. Coleman GP, Fevre ME, and Cleaveland S. Estimating the public health impact of rabies-vol10 no 1 January 2004

3. World Health Organization strategies for the control and elimination of rabies in Asia.Report of a WHO integral consultation. Geneva: The organization 2002.

4. Sudarshan MK, Madhusudana SN, Mahendra BJ, et al. Assessing the burden of human rabies in India: results of a national multi-center epidemiological survey. Int J Infect Dis 2007; 11: 29-35.

5. Hampson K, Dobson A, Kaare M, et al. Rabies exposures, post-exposure prophylaxis and deaths in a region of endemic canine rabies. PLoS Neg1 Trop Dis 2008; 2: e339.

6.Knobel DL, Cleaveland S, Coleman PG, et al (2005). Re-evaluating the burden of rabies in Africa and Asia.

7. Murray CJL, Lopes AD. The global burden of diseases: a comprehensive assessment of mortality and disability from diseases, injuries and risk factors in 1990 and projected to 2020. Cambridge: Harvard University press 1996.
8. Mallewa M, Fooks AR, Banda D, et al Rabies encephalitis in malaria-endemic areas, Malawi, Africa. Emerg.infect.diseas 2007; 13:136-139

9. Gsell AS (2006) Demographic, spatial and behavioural heterogeneities in an urban dog (Canis familiaris) population, relevant in planning rabies control in Iringa, United Republic of Tanzania. MSc thesis, Swiss Tropical Institute, University of Basel

10. Dodet B, The Asian Rabies Expert Bureau. Preventing the incurable: Asian rabies experts advocate rabies control. Vaccine 2006; 24: 3045-3049

11. Kaare M, Lembo T, Hampson K, et al. Rabies control in rural Africa: evaluating strategies for effective domestic dog vaccination. Vaccine 2009; 27: 152-160

12. Cleaveland S, Kaare M, Tiringa P, Mlengeya T, Barrat J (2003) A dog rabies vaccination campaign in rural Africa: impact on the incidence of dog rabies and human dog-bite injuries.

13.Knobel DL, Cleaveland S, Coleman PG, et al. Reevaluating the burden of rabies in Africa and Asia. Bull World Health Organ 2005; 83: 360-368.

14. World Health Organization (2004) Disease and injury regional estimates for 2004. Available at: http://www.who. int/healthinfo/global_burden_disease/estimates_regional/ en/index.html. Accessed 23/3/09.

15.World Health Organization (2003) Cumulative number of reported probable cases of severe acute respiratory syndrome (SARS), epidemic and pandemic alert and response. Available at: http://www.who.int/csr/ sars/country/table2004_04_21/en/index.html. Accessed 25/3/09.

16.World Health Organization (2008) Cumulative number of confirmed human cases of avian influenza $\mathrm{A}$ / (H5N1) reported to WHO. Available at: http://www. who.int/csr/disease/avian_influenza/country/cases_ table_2008_02_21/en/index.html. Accessed 23/3/09.

17. World Health Organization (2001) Nipah virus, Fact Sheets. Available at: http://www.who.int/mediacentre/ factsheets/fs262/en/.

18. World Health Organization (2007) Rift Valley Fever in Kenya, Somalia and the United Republic of Tanzania. Disease Outbreak News, 9 May 2007, http://www.who. int/csr/don/2007_05_09/en/index.html.

19. World Health Organization (2008) Rift Valley Fever in Sudan. Disease Outbreak News, 22 January 2008, http:// www.who.int/csr/don/2008_01_22/en/index.html 